\title{
La educación emocional como método preventivo para el acoso escolar en educación infantil: Una revisión sistemática de la literatura
}

Mónica Girón Barrenengoa - Universidad de Valladolid Manuel Carabias Herrero - Universidad de Valladolid Ana Martín Romera - Universidad de Valladolid

Recepción: 03.12.2020 | Aceptado: 03.02.2021

Correspondencia a través de ORCID: Ana Martín Romera

0000-0002-4805-1849

0000-0002-0436-0738

(D) $0000-0002-2895-5891$

Citar: Girón Barrenengoa, M, Carabias Herrero, M y Martín Romera, A (2021). La educación emocional como método preventivo para el acoso escolar en educación infantil: Una revisión sistemática de la literatura. REIDOCREA, 10(6), 1-15. [ ]

Resumen: Dentro de los contenidos que se desarrollan en la etapa de Educación Infantil, se debe promover y desarrollar la educación emocional y sus distintas formas de expresión de todo el alumnado, entendiendo que supone una medida de prevención para gestionar casos de acoso escolar. Este trabajo es el resultado de un Trabajo Fin de Grado que tuvo como objetivo principal analizar si se trabajaba la educación emocional en las aulas de educación infantil para prevenir el acoso escolar, con la finalidad de derivar pautas de intervención. Para ello se realizó una revisión sistemática de la literatura en Dialnet, en el periodo de 2010 al 2019. Según los estudios analizados se puede concluir que, a pesar de la importancia de estos dos temas, en los últimos años si se está trabajando la educación emocional en la etapa de Educación Infantil pero sin estar directamente relacionado con la prevención del acoso escolar, teniendo una mayor presencia dicha relación en la etapa de Educación Primaria. A partir del análisis de las evidencias en ésta se definen posibles propuestas para trabajar dicha relación en educación infantil.

Palabras clave: Acoso

Emotional education as a preventive method for bullying in early childhood education: A systematic review

Abstract: Among the contents that are developed in Early Childhood Education, emotional education and its different expression must be promoted, understanding that it is a preventive measure to manage bullying. The main purpose of this work was to analyze whether emotional education was worked in children's education classrooms to prevent bullying and deriving intervention guidelines. For this, a systematic review on Dialnet was carried out (period from 2010 to 2019). These two issues do not work in Early Childhood Education, but without being directly related to the bullying prevention, having such a greater presence in Primary Education. Based on the analysis of the evidence in it, possible proposals for working on this relationship in early childhood education are defined.

Keywords: Bullying

\section{Introducción}

En la actualidad, la violencia o la agresividad en el ámbito escolar es un tema de gran relevancia e importancia. En los estudios realizados por la Asociación Mundial de Educadores Infantiles, (World Asociación of Early Chilhood Educators, WAECE), como es el caso del estudio sobre el acoso escolar en la etapa de Educación Infantil, denominado "Estudio Cisneros X" (2007), se pone de manifiesto la afirmación de que el abuso escolar se gesta en esta etapa educativa, ya que los niños, sobre todo los de cinco y seis años, manifiestan conductas y comportamientos que posteriormente serán importantes para las siguientes etapas educativas. Es en estas edades donde comienzan a manifestar signos y evidencias claras que nos pongan en alerta y evidencien un caso de abuso escolar.

El acoso escolar está presente en todos los centros escolares, afectando a niño/as sin importar el ámbito social en el que vivan. En España (Fundación ANAR, 2018) se 
identifica claramente un aumento del porcentaje de casos de bullying, en niños adolescentes en la mayoría de las comunidades autónomas. Los porcentajes de acoso escolar se sitúan en un $53{ }^{\prime} 2 \%$ en el género masculino, y un $46{ }^{\prime} 8 \%$ en el género femenino. Las edades más comunes en las que se produce este fenómeno se sitúan en 7 años (14,7\%), 12 años (13,7\%) y 13 años (13,1\%). Siendo casi la mitad de las víctimas $(43,7 \%)$ menores de 11 años.

El acoso escolar en la etapa escolar puede producir tres tipos de consecuencias según dicha fundación: amonestación de los profesores a las víctimas, cambio de centro escolar y tratamiento psicológico. Con respecto a estos $-y$ por su relación con la educación emocional-, en el año 2015, el síntoma más predominante era la ansiedad con 79 '5\%, en cambio en el año 2016 , destacan los síntomas depresivos con un 72 '2\%. Los problemas que surgen con menos frecuencia son los trastornos de sueño, las diferencias con los profesores y el absentismo, aunque este último aumenta a un $6{ }^{\prime} 3 \%$ con respecto al año anterior.

Desde la escuela y junto con la familia, se debe actuar y prevenir, ya que son los ámbitos más importantes donde el menor se relaciona y se crea como ser social. Es por ello, que entendemos importante crear un buen desarrollo socio afectivo y emocional desde edades tempranas.

Los centros educativos están incluyendo la educación en materia de inteligencia emocional en sus aulas a través de proyectos y distintas iniciativas, se está demostrando que la inteligencia emocional produce efectos positivos en los alumnos, es igual de importante lo que aprenden a través de los distintos aprendizajes como lo que sienten cuando lo logran, se produce entre otras, una mejora en su conducta, se fomentan el aprendizaje, la responsabilidad, la transmisión de sus sentimientos a través de un buen canal de comunicación etc. En conclusión, se produce un buen desarrollo integral del niño, no solo se debe trabajar con el ámbito de la inteligencia y de los conocimientos, sino que también hay que trabajar y educar los comportamientos de los alumnos para lograr un buen desarrollo social (Bisquerra, 2000, 2012), aspecto que viene recogido en el currículo de esta etapa educativa (Real Decreto 1630/2006, de 29 de diciembre).

La educación emocional proporciona efectos positivos en los individuos, según Bisquerra (2006), se produce un aumento de las habilidades sociales y de las relaciones interpersonales, se disminuyen los pensamientos negativos, las conductas violentas y las agresiones, en definitiva, se produce una mejora de la autoestima y de los comportamientos, ya que en el entorno escolar, la educación emocional evita y disminuye las expulsiones que se producen en el aula y aumenta su rendimiento escolar.

Es importante que el profesorado tenga información y esté formado para tratar el tema, observando y sabiendo cuáles suelen ser las características más comunes de los niños que son más vulnerables a sufrirlo, así como también de los "agresores", ya que en el caso de observar esas características, a través de diferentes conductas violentas, que pueden ser tanto físicas como verbales, deberá saber cuáles son las pautas que debe seguir para intervenir, encontrando la base del problema y actuando de diferentes maneras.

Por lo tanto, desde el ámbito educativo, así como el familiar y social, es conveniente y casi obligatorio ayudar y ofrecer a los niños los instrumentos y herramientas necesarias que les ayuden a identificar sus sentimientos y emociones, lo que sienten y cómo esto se refleja en sus actitudes, en cómo les afecta y proponer alternativas para que lo puedan gestionar. En conclusión, hay que educar emocionalmente a nuestros alumnos, a fin de que se conviertan en personas emocionalmente inteligentes, que entiendan sus 
sentimientos y los de los demás, con una autoestima positiva, niños con un bajo nivel de frustración, en definitiva, felices.

Uno de los aspectos sobre los que se puede intervenir es la educación emocional, la cual está teniendo mucha repercusión en la docencia y en el aprendizaje de los alumnos en la actualidad. Relacionado con ello, un estudio reciente (Muñoz-Prieto, 2017) evidencia la relación que existe entre el acoso escolar y la educación emocional. Los alumnos con buenas competencias emocionales, es decir, buen nivel de autoestima, capacidad de empatizar, buenas habilidades sociales, mejor conocimiento de sí mismos, que saben gestionar sus emociones y las de los demás, suelen ser capaces de evitar o gestionar casos de acoso escolar. Así, una búsqueda inicial sobre la relación entre aspectos mostró que en los últimos años se han venido desarrollando trabajos académicos (Trabajos de Fin de Grado) tales como "El acoso escolar: propuesta de intervención a través de la educación emocional y en valores" (Izquierdo, 2017), o "Prevención del acoso escolar en educación infantil: propuesta de educación emocional para la resolución pacífica de conflictos" (Mosquera, 2015). Es por este motivo por lo que nos interesamos en averiguar qué relación existe entre estos dos términos, analizando el modo en que se trabaja en Educación Infantil. Atendiendo a ello, este trabajo presenta los resultados de una revisión sistemática de la literatura sobre los trabajos existentes al respecto, considerando que puede ayudar a derivar pautas de actuación para esta etapa educativa.

Con la finalidad de derivar pautas de intervención para prevenir el acoso escolar a través de la educación emocional, es necesario realizar un estudio exhaustivo de los trabajos que se han realizado hasta el día de hoy. Este trabajo, que se deriva de un Trabajo Fin de Grado, fue realizado a través de una revisión sistemática de la literatura, interesándose en averiguar qué relación existe entre estos dos términos, analizando el modo en que se trabaja en Educación Infantil.

\section{Objetivos o hipótesis}

La finalidad de este trabajo es analizar cómo se trabaja la educación emocional en el aula para prevenir el acoso escolar en la etapa de educación infantil, derivando pautas de intervención.

Para la consecución de esta finalidad, se propuso como objetivos los siguientes:

- Averiguar qué relación existe entre la educación emocional y el acoso escolar en educación infantil.

- Dar a conocer cómo se trabaja la educación emocional para disminuir el acoso escolar.

\section{Métodos}

Se ha realizado un estudio siguiendo la metodología propia de las revisiones sistemáticas de la literatura (Beltrán, 2005). Se trata de "un estudio integrativo, observacional, retrospectivo, secundario, en el cual se combinan estudios que examinan la misma pregunta" (p. 62). En este trabajo se emplea una revisión sistemática "cualitativa u overview", tomando como base los siguientes pasos (Beltrán, 2005) (Ver Figura 1): 
Figura 1. Proceso de revisión sistemática

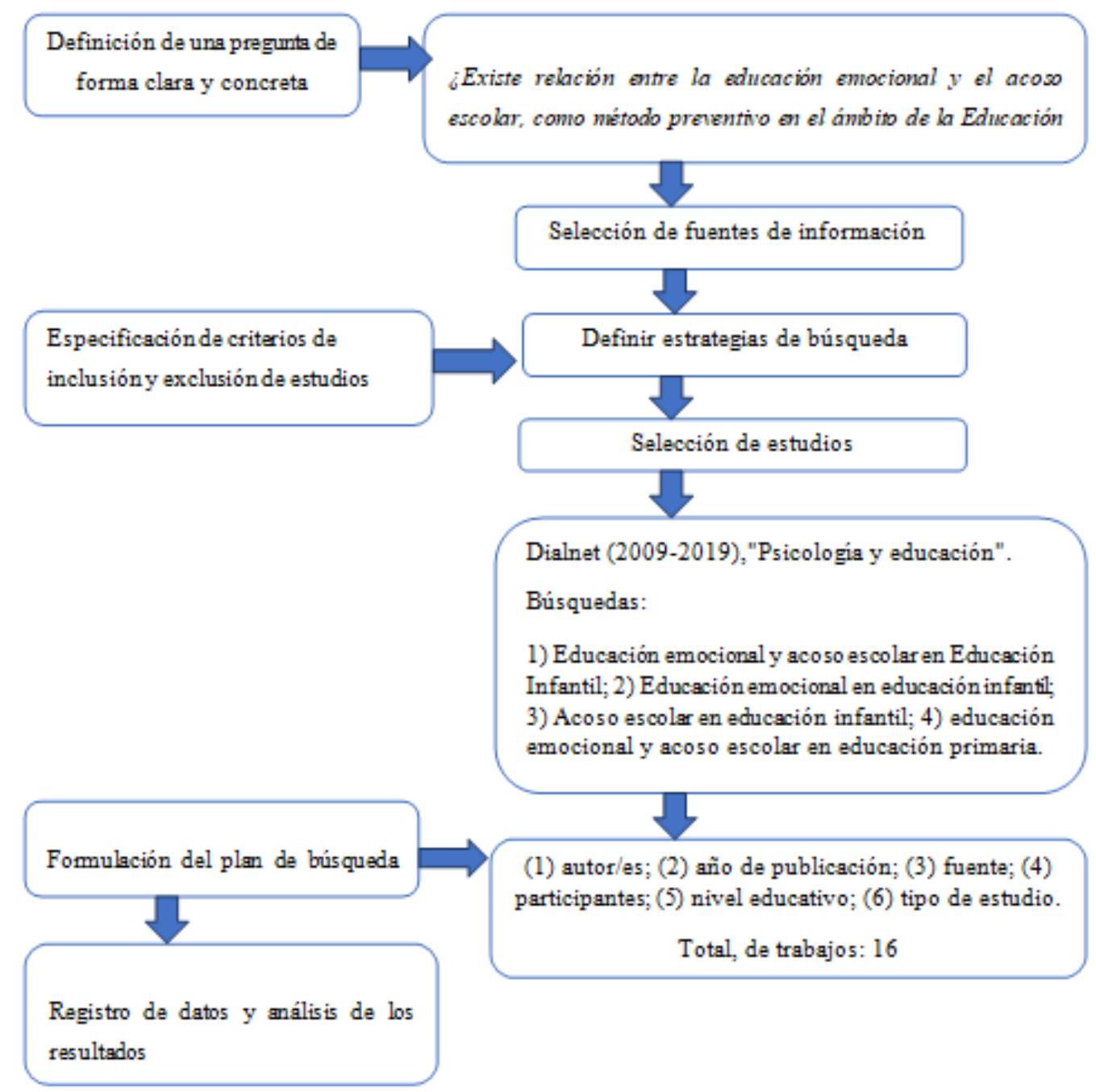

Nota. Elaboración propia a partir de Beltrán (2005).

1. Definir una pregunta claramente, a qué pregunta se quiere responder.

En este trabajo se ha realizado una revisión bibliografía, en revistas científicas, tesis, artículos, para dar respuesta a la siguiente pregunta de investigación: ¿Existe relación entre la educación emocional y el acoso escolar, como método preventivo en el ámbito de la Educación Infantil?

2. Especificación de los criterios de inclusión y exclusión de documentos.

Los criterios de inclusión que se han llevado a cabo han sido: "idioma" y "contexto", "periodo de revisión", "materias" y "tipo de trabajo". Estos fueron concretados de la siguiente forma: idioma y contexto español, periodo de revisión referido a nueve años, materia Dialnet Piscología y Educación, y tipos de estudios teóricos, empíricos, y programas y experiencias educativas 
Se decidió realizar la búsqueda en un periodo de diez años, debido a la actualidad e importancia que el tema ha adquirido en los últimos años, por este motivo también se decidió a hacerlo en el idioma y contexto español, a través de estudios empíricos que diesen validez a los objetivos que se pretendían alcanzar en el trabajo.

Se incluyó como criterio de inclusión la "etapa educativa", en este caso "educación infantil". Así, tras una primera búsqueda atendiendo a este criterio los resultados no fueron satisfactorios. No se encontraron resultados directamente relacionados con dicha etapa, ampliando el criterio de búsqueda a "educación primaria", por ser esta la etapa más cercana a la etapa de educación infantil, entendiendo que los resultados podrían trasladarse a dicha etapa.

\section{Formulación del plan de búsqueda de la literatura.}

Para realizar la búsqueda se utilizó la base de datos Dialnet, ya que esta recoge más 1000 revistas en el área de psicología y educación, por lo que nos permitió acceder a un amplio estudio sobre la temática seleccionada. El periodo de esta búsqueda se ha centrado en los anteriores 10 años (2009-2019), la cual fue realizada en el mes de mayo de 2019, atendiendo a la materia Dialnet de "Psicología y Educación".

Empleamos los siguientes criterios de búsqueda, palabras clave utilizadas, empleando los términos "educación emocional", "acoso escolar" y "educación infantil" de forma conjunta. De forma inicial, la búsqueda realizada fue "educación emocional y acoso escolar en educación infantil", obteniendo 7 documentos, tal como se muestra en la Figura 2.

Encontramos trabajos referidos a educación primaria y educación secundaria obligatoria, y un trabajo que atendía a educación infantil: "Cómo promover la convivencia: Programa de Asertividad y Habilidades Sociales (PAHS): (Educación Infantil, Primaria y Secundaria)" dirigido por Monja Casares, y firmado por de Benito Pascual, Elices Simón, Francia Conde y García Larrauri (2009). En este documento se habla sobre los problemas de convivencia que se producen en las aulas y el contexto escolar y que métodos se deben utilizar para afrontar y dar solución a estos problemas, entre ellos se destaca la formación del profesorado y programas de enseñanza en habilidades para una correcta convivencia, desarrollando vínculos emocionales, respeto y actitudes positivas. Es por ello, que se decidió realizar una búsqueda pormenorizada de los conceptos.

Se procedió a realizar una búsqueda de documentos relacionados con los conceptos a tratar, es decir, "educación emocional" y "acoso escolar", por separado, buscando individualmente documentos que expliquen la relación que existe entre los dos conceptos y el modo en que se trabaja en Educación Infantil. 
Figura 2. Captura de pantalla de búsqueda inicial de literatura en Dialnet

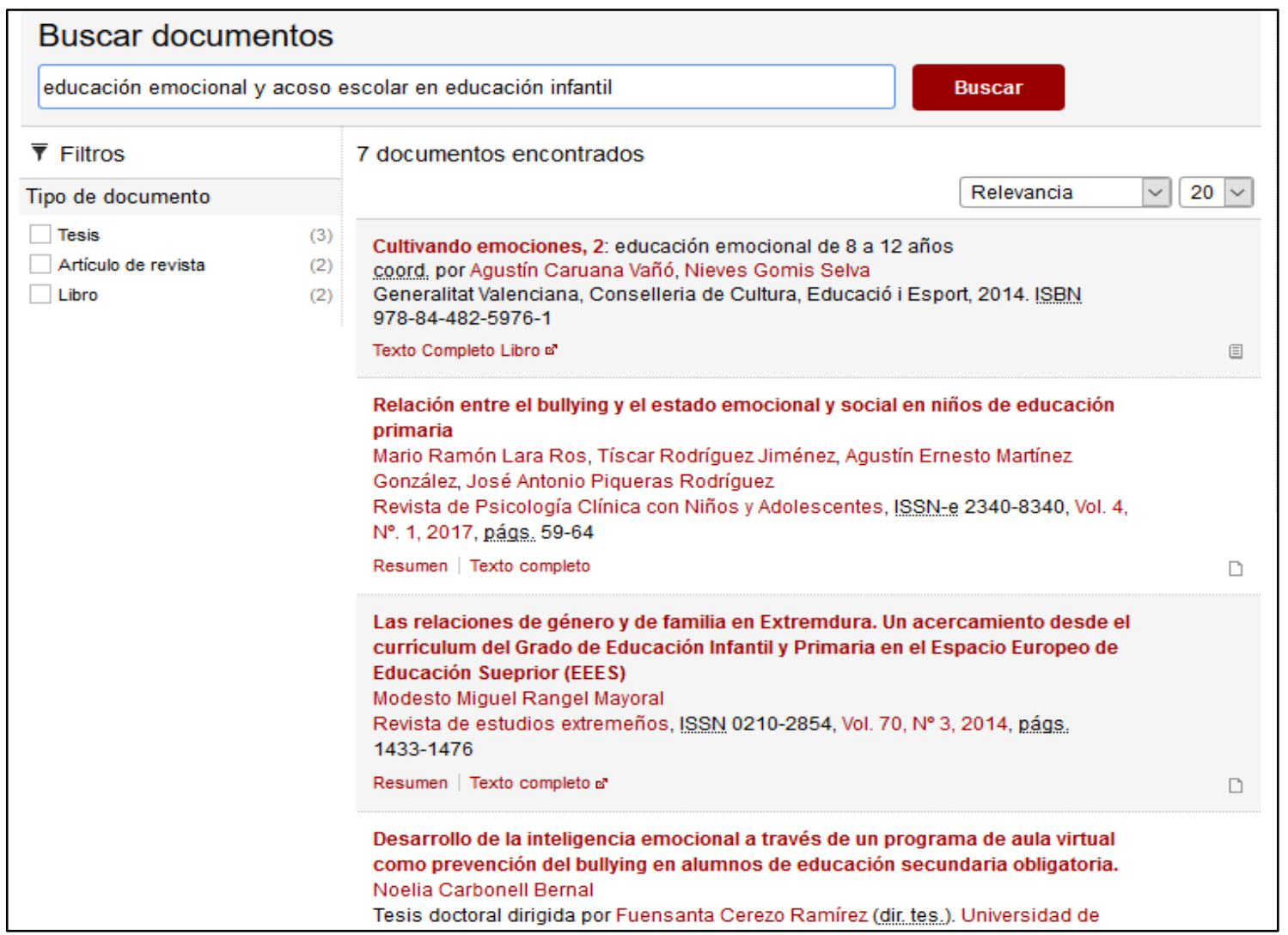

Una segunda búsqueda atendió a los términos "educación emocional" y "educación infantil" (Figura 3). Se decidió realizar una búsqueda sobre el concepto de educación emocional en la etapa de educación infantil, obteniendo 26 resultados, entre los cuales se destaca "Educación emocional en los profesores de Educación Infantil aspecto clave en el desempeño docente" (Sáenz de Jubera Ocón y Luis Rico, 2017), el cual trata sobre la importancia de que los profesores de la etapa de educación infantil estén formados en educación emocional, ya que a través de un estudio realizado en centros educativos de Burgos y Miranda de Ebro, se llegó a la conclusión de que un 69'9\% de los cursos escolares tienen relación con la educación emocional o inteligencia emocional, habiéndose triplicado el tratamiento de este tema en los últimos años en los centros públicos. Por lo tanto, es un aspecto fundamental que los docentes estén formados en el tema para que puedan llevar a cabo metodologías activas que faciliten el desarrollo integral de los alumnos. 
Figura 3. Captura de pantalla de búsqueda sobre educación emocional en la etapa de educación infantil en Dialnet

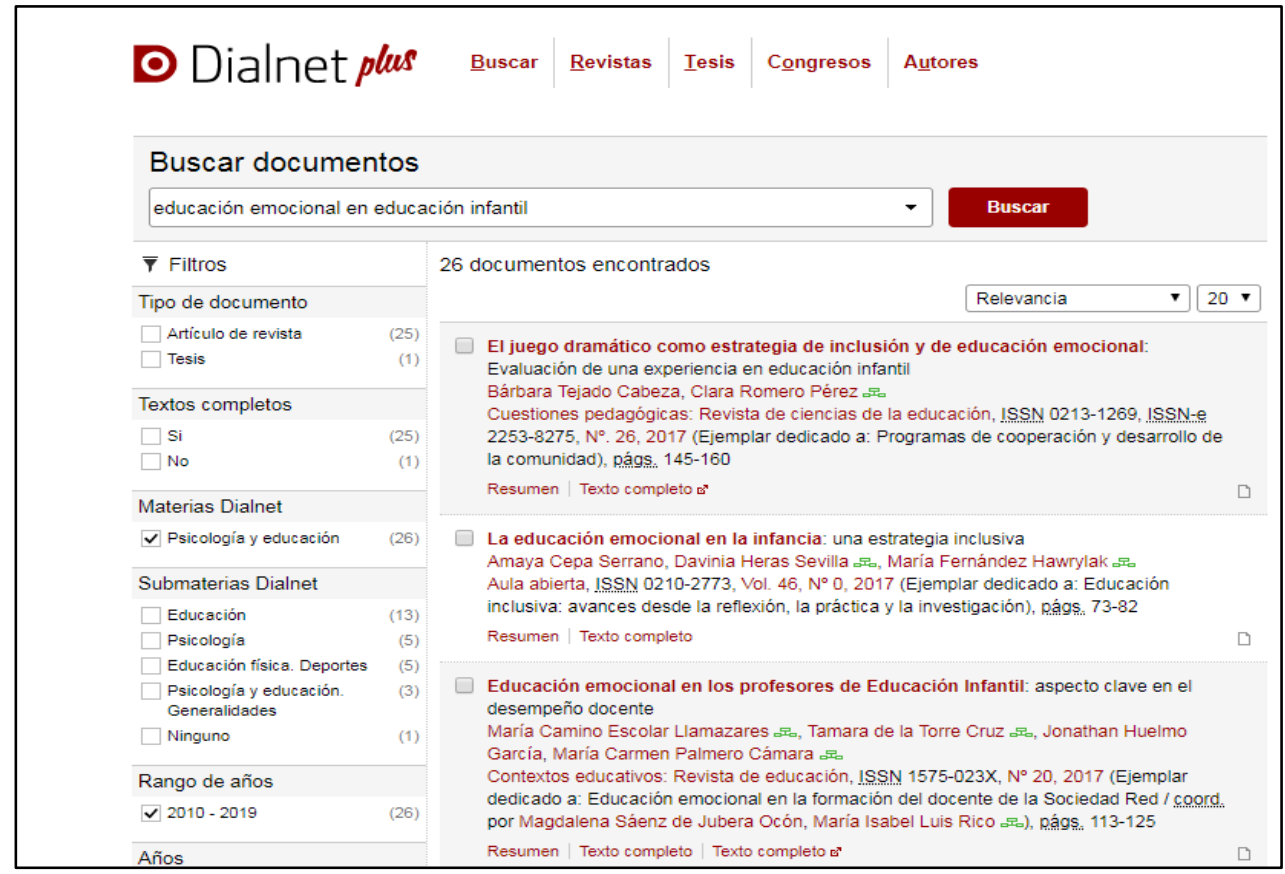

En búsqueda posterior de "acoso escolar" y "educación infantil, se obtuvieron 18 resultados (Figura 4). Cabe destacar que en ellos no aparecía el concepto relacionado directamente con la etapa seleccionada, si no que los documentos nos derivaban principalmente a casos relacionados con la etapa de educación primaria o educación secundaria.

Figura 4. Captura de pantalla de búsqueda sobre acoso escolar en la etapa de educación infantil en Dialnet

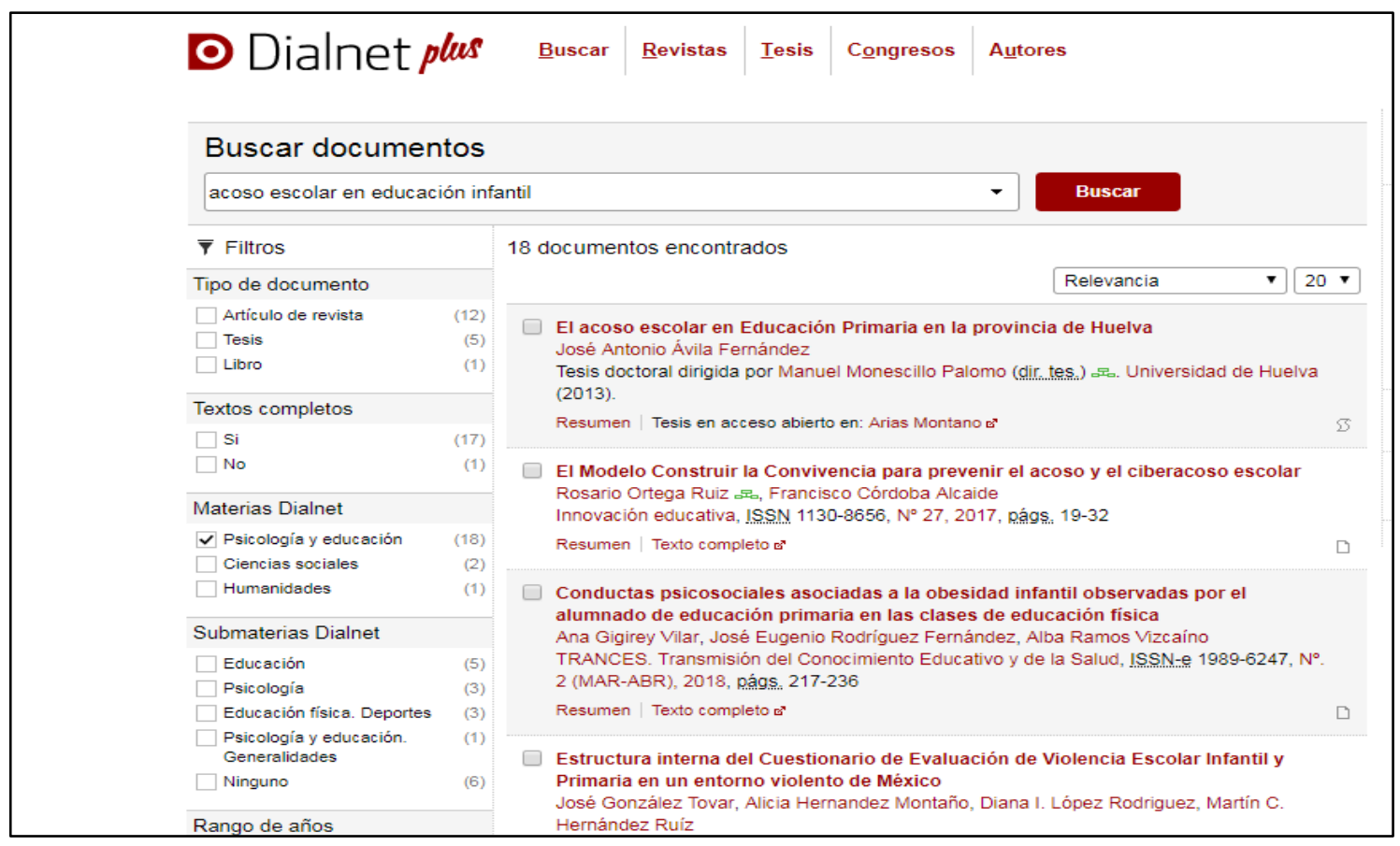


Al no obtener muchos datos centrados en el ámbito de la educación infantil sobre los dos términos seleccionados, se decidió ampliar el campo hacia el ámbito de la "educación primaria", buscando estos dos términos conjuntamente: "educación emocional y acoso escolar en educación primaria". La búsqueda nos mostró 11 documentos (Figura 5). Entre ellos destacamos una propuesta de intervención denominada "Agresividad entre iguales: detección de necesidades y propuesta de intervención educativa para mejorar la convivencia escolar en un aula de educación primaria" (Domínguez, 2015). En este documento se trata el tema de la agresividad entre iguales, las características de los implicados y las causas y consecuencias que produce el acoso escolar en las víctimas, dando importancia a la educación emocional como estrategia principal para prevenir el acoso escolar.

Una vez que se realizaron las búsquedas comentadas, se procedió a una lectura de los trabajos que atendían a las necesidades de la pregunta propuesta, de acuerdo a un conjunto de criterios de inclusión y exclusión para cada búsqueda.

\section{Instrumento}

Para realizar en análisis y extraer los datos, se creó una plantilla de Excel en la que se plasmaron los documentos más relevantes que se habían obtenido en las búsquedas, obteniendo así un estudio que nos diera validez y que fuese actual. Para ello, en la tabla elaborada se añadieron datos significativos, con las siguientes variables: (1) autor/es; (2) año de publicación; (3) fuente; (4) participantes; (5) nivel educativo; (6) tipo de estudio.

Figura 5. Captura de pantalla de búsqueda sobre los conceptos de educación emocional y acoso escolar en la etapa de educación primaria en Dialnet

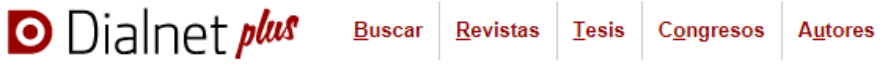

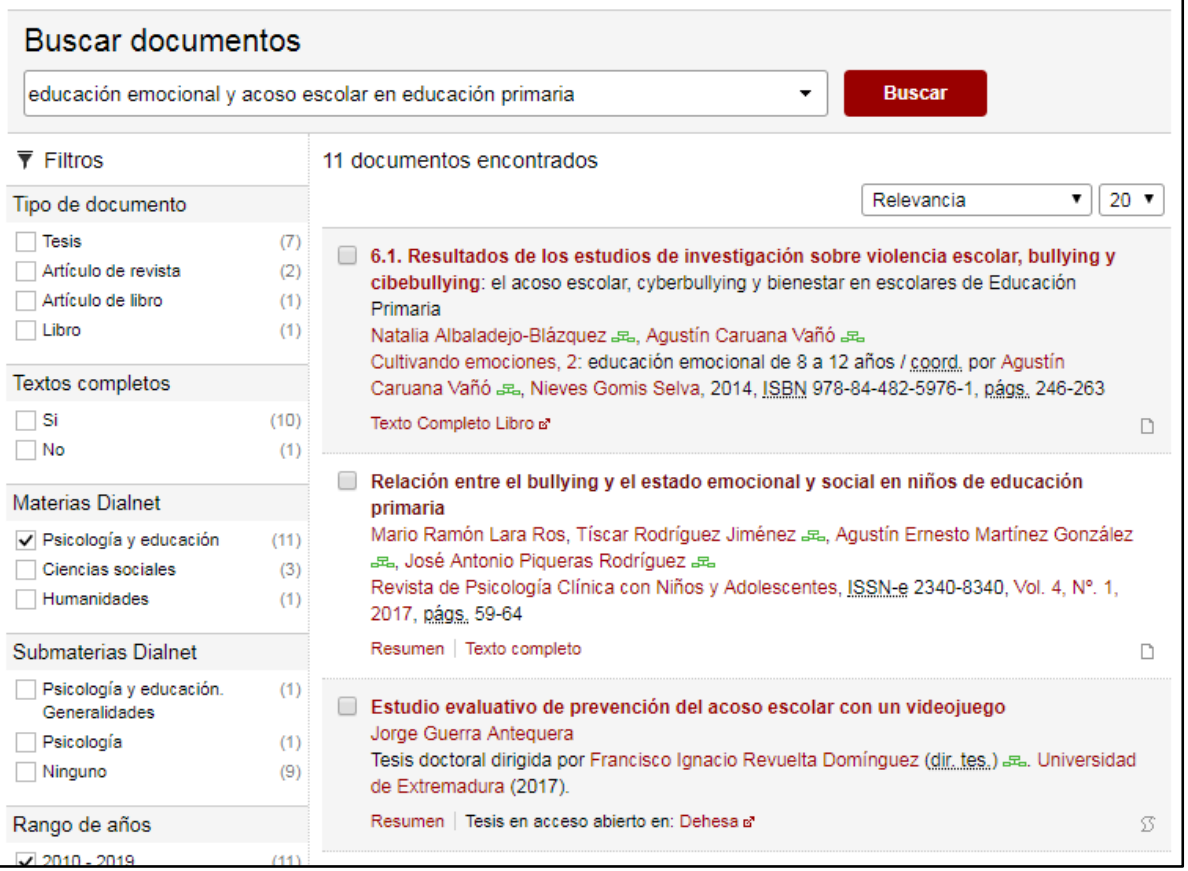




\section{Resultados}

En este apartado se pueden observar los estudios que han sido seleccionados para realizar la revisión sistemática, los cuales se recogen y se detallan en la Tabla 6.

En la siguiente tabla se han seleccionado un total de 16 documentos, entre los cuales seis están directamente relacionados con la educación emocional y el acoso escolar en educación primaria (indicados con color naranja). Son estudios de investigación, realizados recientemente, ya que sus fechas están comprendidas entre los años 2009 y 2017.

El análisis de los documentos seleccionados nos muestra los siguientes resultados:

Realizando la búsqueda sobre "educación emocional y acoso escolar en educación infantil", no encontramos resultados directamente relacionados, pero sí se obtienen resultados atendiendo a los conceptos por separado. En ellos, observamos que en este ámbito se realizan programas de intervención y propuestas metodológicas para abordar la educación emocional, a través de un desarrollo globalizado que tenga en cuenta las características individuales del alumnado. Esto lo podemos encontrar en el trabajo "Mejora de la inteligencia emocional en educación infantil y desarrollo cognitivo" (Gelabert Amengual, 2014), en el que crea un programa de intervención, teniendo en cuenta los procesos psicológicos básicos que describieron Mayer y Salovey (1997) para el desarrollo de la inteligencia emocional, adaptando el currículo de la etapa de educación infantil.

Sin embargo, en el ámbito de la educación primaria, se encuentran 6 trabajos relacionados con los términos seleccionados, que nos informan sobre la relevancia que el acoso escolar tiene en educación infantil y que ponen de manifiesto que este se manifiesta cada vez más en edades más tempranas. A través de los resultados evidencia la importancia que tiene trabajar la educación emocional en educación infantil para prevenir el acoso escolar.

En el trabajo "Percepción y atribución causal sobre el bullying y sus efectos emocionales en escolares de primaria" (Rodríguez Barbero Torres, 2015), se pone de manifiesto que en educación primaria es importante la expresión gráfica para detectar posibles casos de acoso escolar, sobre todo el dibujo. A través de éste, los niños expresan sus sentimientos, sus opiniones y actitudes con respecto a fenómenos sociales que sin este método de expresión serian difíciles de conocer en esta etapa. Esto se debe a que el niño no tiene la suficiente capacidad léxica para expresar a través del lenguaje y de la comunicación lingüística todo lo que siente, como lo hace realmente con el dibujo.

En la tesis "Agresividad entre iguales: detección de necesidades y propuesta de intervención educativa para mejorar la convivencia escolar en un aula de educación primaria" (Domínguez, 2015), surge la necesidad por solucionar los problemas que se producen en las aulas por falta de disciplina. Se realiza un estudio empírico con el objetivo de conocer y analizar el clima que se produce a través de la convivencia escolar en un aula de $4^{\circ}$ de primaria. Observando qué situaciones de agresividad se producen y qué necesidades se encuentran, se diseña un taller de convivencia, a través de la técnica didáctica de dinámica de grupos: trabajos en grupo, en equipos, lluvias de ideas, etc., identificando sus emociones e interpretando sus estados de ánimo y los de los demás. También se utiliza la técnica del role-play, para realizar las conductas de los diferentes miembros de los grupos y analizarlas. Todo esto pretende dar solución a las necesidades que se plantean, evaluando la posibilidad de mejorar el ambiente del aula para disminuir las situaciones agresivas entre iguales. 
El libro "Cultivando emociones 2, educación emocional de 8 a 12 años, cuyos coordinadores son Agustín Caruana Vañó y Nieves Gomis Selva (2014), posee un apartado (1.2), que trata sobre la violencia escolar. Se afirma la importancia de trabajar y abordar el tema de las emociones en el ámbito educativo, ya que ayudan a mejorar la convivencia en el aula y prevenir conductas violentas. Desarrollan un programa de educación emocional y prevención de la violencia dirigido a la ESO, a través de las siguientes estrategias (p. 44), coincidiendo con los dos trabajos citados anteriormente:

- Enfoque preventivo inespecífico de la violencia escolar promoviendo la educación emocional.

- Propuesta de actuación a través del plan de acción tutorial.

- Utilización de actividades vivenciales, dinámicas y participativas (enfoque socioafectivo).

- Utilización de actividades de bajo coste en cuanto a los materiales necesarios (de lápiz y papel, etc.) y fácil ejecución, previamente testadas en las aulas.

- Agrupación y organización de las actividades en módulos según las competencias emocionales que contribuyen a desarrollar: autoconocimiento, autocontrol, habilidades sociales, resolución de conflictos, empatía, etc.

- Impulso de estrategias metodológicas inclusivas mediante la formación de grupos heterogéneos y fomentando el trabajo cooperativo.

- Inclusión de los resultados de estudios de investigación con alumnado.

Encontramos un trabajo denominado "Relación entre el bullying y el estado emocional y social en niños de educación primaria" (Lara Ros et al., 2017), tenía como objetivo principal analizar qué relación mantenían el bullying, el acoso escolar y los síntomas ansiosos y depresivos que se producían en víctimas de 9 a 12 años. El estudio mostró que un $17 \%$ de los alumnos estaban relacionados e implicados con casos de bullying, mostrando que las víctimas, mayoritariamente de sexo femenino, tenían altos niveles de síntomas ansiosos y depresivos. Este trabajo, al igual que los anteriores, concluyen que las situaciones de violencia se producen en edades tempranas, iniciándose antes de la adolescencia, al igual que la importancia de crear y llevar a cabo programas de prevención que ayuden a desarrollar habilidades de interacción social y de intervención en el ámbito escolar, dirigido a toda la comunidad educativa. 
Tabla 1. Resultados obtenidos de la búsqueda realizada

\begin{tabular}{|c|c|c|c|c|c|}
\hline TITULO & AUTOR/ES & AÑO & FUENTE & $\begin{array}{c}\text { NIVEL } \\
\text { EDUCATIVO } \\
*\end{array}$ & $\begin{array}{l}\text { TIPO DE } \\
\text { ESTUDIO** }\end{array}$ \\
\hline $\begin{array}{l}\text { Como promover la convivencia. Programa } \\
\text { de asertividad y habilidades sociales } \\
\text { (PAHS): educación infantil, primaria y } \\
\text { secundaria. }\end{array}$ & de Benito Pascual et al. & 2009 & Editorial CEPE & Ed.I/Ed.P/Ed.S & $\mathrm{T}$ \\
\hline $\begin{array}{l}\text { Desarrollar la inteligencia emocional para } \\
\text { prevenir el acoso escolar. }\end{array}$ & Muñoz Prieto & 2017 & $\begin{array}{l}\text { Revista Escuela } \\
\text { Abierta }\end{array}$ & $\mathrm{N}$ & $\mathrm{T}$ \\
\hline $\begin{array}{l}\text { La educación emocional en la Educación } \\
\text { Infantil y Primaria }\end{array}$ & López & 2016 & $\begin{array}{ll}\text { Capítulo } & \text { de } \\
\text { libro } & \text { en } \\
\text { Inteligencia } & \\
\text { Emocional } & \text { y } \\
\text { Bienestar II. } & \\
\end{array}$ & Ed. P/Ed. I & $\mathrm{T}$ \\
\hline $\begin{array}{l}\text { Educación emocional en la formación del } \\
\text { docente de la Sociedad Red. }\end{array}$ & Sáenz de Jubera Ocón y Luis Rico & 2017 & $\begin{array}{l}\text { Contextos } \\
\text { Educativos }\end{array}$ & Ed. I & $\mathrm{T}$ \\
\hline $\begin{array}{l}\text { Agresividad entre iguales: detección de } \\
\text { necesidades y propuesta de intervención } \\
\text { educativa para mejorar la convivencia } \\
\text { escolar en un aula de educación primaria }\end{array}$ & Domínguez & 2015 & Tesis Doctoral & Ed. P & $E, P$ \\
\hline $\begin{array}{l}\text { Cultivando emociones } 2, \quad \text { educación } \\
\text { emocional de } 8 \text { a } 12 \text { años }\end{array}$ & Caruana Vañó y Gomis Selva & 2014 & $\begin{array}{l}\text { Conselleria de } \\
\text { Cultura, } \\
\text { Educación i } \\
\text { Esport }\end{array}$ & Ed. P & $\mathrm{T}$ \\
\hline $\begin{array}{l}\text { Relación entre el bullying y el estado } \\
\text { emocional y social en niños de educación } \\
\text { primaria }\end{array}$ & Lara Ros et al. & 2017 & $\begin{array}{lr}\text { Revista } & \text { de } \\
\text { Psicología } & \\
\text { Clínica con } \\
\text { Niños y } \\
\text { Adolescentes }\end{array}$ & Ed. P & $E$ \\
\hline
\end{tabular}




\begin{tabular}{|c|c|c|c|c|c|}
\hline $\begin{array}{l}\text { Formación inicial del profesorado de } \\
\text { Educación Infantil: claves para el diseño } \\
\text { innovador de planes de estudio }\end{array}$ & Domínguez Garrido & 2015 & $\begin{array}{ll}\text { Revista } & \\
\text { Facultad } & \text { de } \\
\text { Educación de } \\
\text { Albacete, }\end{array}$ & Ed. I & E \\
\hline $\begin{array}{l}\text { Educación emocional en los profesores de } \\
\text { educación infantil aspecto clave en el } \\
\text { desempeño docente }\end{array}$ & Escolar Llamazares et al. & 2017 & $\begin{array}{l}\text { Revista de } \\
\text { Educación }\end{array}$ & Ed. I & E \\
\hline $\begin{array}{l}\text { Importancia de la inteligencia emocional en } \\
\text { la práctica docente: un estudio con } \\
\text { maestros }\end{array}$ & Cejudo y López-Delgado & 2017 & $\begin{array}{l}\text { Revista } \\
\text { Psicología } \\
\text { Evolutiva }\end{array}$ & Ed. I/ Ed. P & E \\
\hline $\begin{array}{l}\text { La educación emocional en la infancia: una } \\
\text { estrategia inclusiva }\end{array}$ & $\begin{array}{l}\text { Cepa Serrano, Heras Sevilla y } \\
\text { Fernández Hawrylak }\end{array}$ & 2017 & $\begin{array}{l}\text { Revista Aula } \\
\text { Abierta }\end{array}$ & Ed. I & E \\
\hline $\begin{array}{l}\text { Mejora de la inteligencia emocional en } \\
\text { educación infantil y desarrollo cognitivo }\end{array}$ & Gelabert Amengual & 2014 & $\begin{array}{l}\text { INFAD Revista } \\
\text { de Psicología }\end{array}$ & Ed. I & $P$ \\
\hline $\begin{array}{l}\text { Resultados de los estudios de investigación } \\
\text { sobre violencia escolar, bullying y } \\
\text { cibebullying: el acoso escolar, cyberbullying } \\
\text { y bienestar en escolares de Educación } \\
\text { Primaria }\end{array}$ & $\begin{array}{l}\text { Albaladejo-Blázquez y Caruana } \\
\text { Vañó }\end{array}$ & 2014 & $\begin{array}{ll}\text { Capítulo } & \text { de } \\
\text { libro } & \text { en } \\
\text { Cultivando } & \\
\text { emociones } 2 & \end{array}$ & Ed. P & $E$ \\
\hline $\begin{array}{l}\text { Percepción y atribución causal sobre el } \\
\text { bullying y sus efectos emocionales en } \\
\text { escolares de primaria }\end{array}$ & Rodríguez Barbero Torres & 2015 & Tesis doctoral & Ed. P & $E$ \\
\hline $\begin{array}{l}\text { Diseño e implementación de un programa } \\
\text { coeducativo para la mejora de la } \\
\text { convivencia y el desarrollo integral de } \\
\text { estudiantes y sus familias en una escuela } \\
\text { de primaria }\end{array}$ & Carell Docal & 2017 & Tesis doctoral & Ed. P & $P, E$ \\
\hline $\begin{array}{l}\text { Análisis ético-descriptivo } \\
\text { comportamiento social del bullying: un } \\
\text { fenómeno epidemiológico susceptible de } \\
\text { ser moldeado matemáticamente para su } \\
\text { prevención e intervención }\end{array}$ & Aranda et al. & 2017 & $\begin{array}{l}\text { Revista Boletín } \\
\text { Redipe }\end{array}$ & & $E$ \\
\hline
\end{tabular}




\section{Discusión}

Este trabajo tuvo como objetivo principal analizar si se trabajaba la educación emocional en las aulas de educación infantil para prevenir el acoso escolar. Para ello se realizó una revisión sistemática de la literatura en Dialnet, en el periodo de 2010 al 2019. Según los estudios analizados se puede concluir que, a pesar de la importancia de estos dos temas, en los últimos años si se está trabajando la educación emocional en la etapa de educación infantil pero sin estar directamente relacionado con la prevención del acoso escolar, ya que en nuestras primeras investigaciones no obtuvimos resultados directamente relacionados con estas dos temáticas. Sin embargo, en las aulas de educación primaria si se trabajan estos dos conceptos relacionados entre sí, ya que el acoso escolar, aunque puede aparecer en la etapa de educación infantil, no es hasta edades posteriores cuando se identifican rasgos característicos de este fenómeno.

A través de algunos trabajos sobre propuestas de intervención hemos podido observar cómo se trabaja con estos dos temas, en los que se llega a la conclusión de que hay que trabajar en las aulas a través de un ambiente relajado, en el que predomine la expresión y la comprensión de los sentimientos e inquietudes, tanto propios como de los demás. Como hemos comprado en el desarrollo de esta investigación, una de las formas de expresión para los niños es el dibujo, a través de este, expresan sus sentimientos y/o sus inquietudes. Otro de los aspectos a destacar es el enfoque socioafectivo, a través de actividades vivenciales, dinámicas y participativas.

La relación entre emociones y bullying se manifiesta evidente, en los casos de bullying tanto los acosadores como las victimas experimentan una serie de emociones, coincidiendo con lo mostrado en el estudio de Muñoz-Prieto (2017). En el caso de los acosadores la experiencia o resultado resulta satisfactorio, pues estamos hablando de una actitud reiterada. En el caso de la víctima se experimentan emociones negativas como ansiedad. Dotar de herramientas a los niños que les permita gestionar sus emociones para que sean capaces de ser conscientes de ellas y de las de los demás, les facilitaría alcanzar niveles de empatía suficientes para detectar, por ejemplo, la ansiedad que otros compañeros sienten por sus actos. Esto es algo que se ha venido demandando desde diversas instancias y estudiosos de este campo (e.g., Bisquerra, 2012; Fundación ANAR, 2018).

El papel que juega el maestro en estas situaciones es fundamental a la hora, no solamente de detectar y aplicar medidas oportunas para detener estas acciones, sino también a la hora de disponer de herramientas que le permitan trabajar con sus alumnos las emociones, actitudes y competencias sociales claves, que eviten en edades superiores que se conviertan en acosadores. En varias investigaciones ha aparecido la formación del profesorado en cuanto a las emociones se refiere, pero no de forma específica a su relación con el acoso escolar. Así, por ejemplo, el trabajo de Mosquera (2015) realiza un conjunto de propuestas para trabajar el acoso escolar, basándose en pautas de intervención emocional, no directamente relacionadas con aquél. No habiendo encontrado trabajos específicos que establezcan en la etapa emocional una relación directa entre ambos aspectos en las propuestas de intervención.

Una vez analizados los documentos encontrados se llega a la conclusión de que debido a la gran relevancia e importancia que estos dos temas están adquiriendo en la actualidad, se podría hacer un propuesta de intervención en un aula de educación infantil, en la que destacaríamos el aspecto socioafectivo y aprovecharíamos recursos tales como el cuento, en los que surjan conflictos entre personajes y como estos lo resuelven, para que a través de ello, los niños adquieran los conocimientos necesarios para poder traspasar lo que surge en el cuento a situaciones cercanas a su vida, ya sea 
tanto en el colegio como en su entorno familiar. Otro de los recursos que utilizaríamos sería el dibujo. Cuando surja un conflicto en el aula o se observe que el niño lo necesita, se realizará un taller de dibujo para que los niños expresen los sentimientos o emociones que estén viviendo en ese momento, ya que es una forma de expresión fácil para ellos, Evitando así que deban manifestar la situación de forma oral, pues es más difícil que lo expresen de esta forma. También es un método de prevención, ya que a través de este se pueden observar rasgos que nos han observar o identificar posibles casos de acoso.

Nos han surgido algunas limitaciones a la hora de realizar este trabajo, una de ellas se refiere a la utilización de base de datos empleadas, centrándonos en Dialnet, aunque hubiese sido necesario e importante haber realizado estas búsquedas en bases de datos internacionales, tales como SCOPUS y Web of Science, nos hubiese permitido haber realizado un estudio más amplio e internacional. Para posibles estudios, se realizarían es dichas bases. Además, al habernos centrado en dicha base de datos, también nos ha limitado las búsquedas, ya que no nos ha permitido acceder por ejemplo a Trabajos de Fin de Grado o Propuestas Didácticas que hay sobre el tema.

\section{Referencias}

Beltran, G (2005). Revisiones sistemáticas de la literatura. Revista Colombiana de Gastroenterología, 20(1), 60-69.

Bisquerra, R (2000). Educación emocional y bienestar. Praxis.

Bisquerra, R (2006). Orientación psicopedagógica y educación emocional. ESE. Estudios sobre educación, 11, 9-25.

Bisquerra, R (2012). De la inteligencia emocional a la educación emocional. En R. Bisquerra (Coord.), ¿Cómo educar las emociones? La inteligencia emocional en la infancia y la adolescencia (pp. 24-35). Hospital de Sant Joan de Déu.

Caruana Vañó, A y Gomis Selva, N (2014). Cultivando emociones 2, educación emocional de 8 a 12 años. Generalitat Valenciana, Conselleria de Cultura, Educació i Esport.

Domínguez Garrido, MC (2015). Formación inicial del profesorado de Educación Infantil: claves para el diseño innovador de planes de estudio. Ensayos: Revista de la Facultad de Educación de Albacete, 30(29), 227-245.

Fundacion ANAR (2018). III Estudio sobre acoso escolar y ciberbullying según los afectados. Informe del Teléfono ANAR. Madrid: Fundación ANAR.

Gelabert Amengual, J (2014). Mejora de la inteligencia emocional en educación infantil y desarrollo cognitivo. International Journal of Developmental and Educational Psychology: INFAD. Revista de Psicología, 2(1), 279-286.

Izquierdo, Al (2014). El acoso escolar: propuesta de intervención a través de la educación emocional y en valores. [Trabajo Fin de Grado, Universidad de Valladolid].

Lara Ros, MR, Rodríguez Jiménez, T, Martínez González, AE y Piqueras Rodríguez, JA (2017). Relación entre el bullying y el estado emocional y social en niños de 
educación primaria. Revista de Psicología Clínica con Niños y Adolescentes, $4(1), 59-64$.

Real decreto 1630/2006 de 29 de diciembre (BOE, №4, Ministerio de educación y ciencia, España 4 de enero de 2007), por el que se establecen las enseñanzas mínimas del segundo ciclo de Educación infantil.

Oñate Cantero, A y Piñuel y Zabala, I (2007). Informe Cisneros X. Acoso y violencia escolar en España. Instituto de Innovación Educativa y Desarrollo Directivo.

Monjas, MI, de Benito Pascual, MP, Elices Simón, JA, Francia Conde, MV y García Larrauri, B (2009). Cómo promover la convivencia: Programa de Asertividad y Habilidades Sociales (PAHS): (Educación Infantil, Primaria y Secundaria. CEPE.

Mosquera, J (2015). Prevención del acoso escolar en educación infantil: propuesta de educación emocional para la resolución pacífica de conflictos. [Trabajo Final de Grado, Universidade de Santiago de Compostela]. Minerva.

Muñoz-Prieto, MM (2017). Desarrollar la inteligencia emocional para prevenir el acoso escolar. Escuela abierta: revista de Investigación Educativa, 20, 35-46.

Rodríguez Barbero Torres, MS (2015). Percepción y atribución causal sobre el bullying y sus efectos emocionales en escolares de primaria. [Tesis Doctoral inédita, Universidad de Córdoba]. Helvia.

Sáenz de Jubera Ocón y Luis Rico, MI (2017). Educación emocional en la formación del docente de la Sociedad Red. Contextos educativos: Revista de educación, 20, 7-9. 